\title{
7 From Quantum Biology Towards Quantum Consciousness
}

\author{
Jack Tuszyński \\ University of Alberta \\ Politecnico di Torino
}

\section{CONTENTS}

Introduction 81

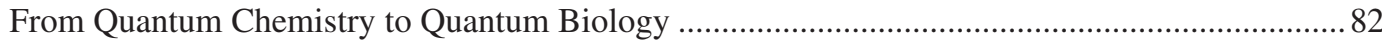

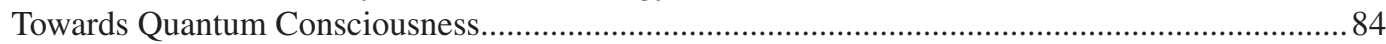

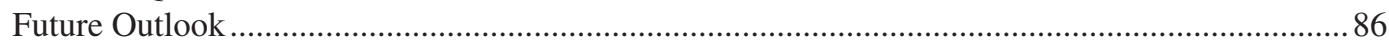

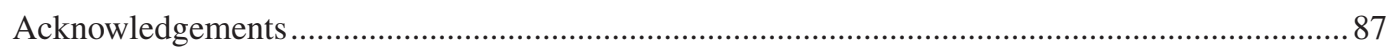

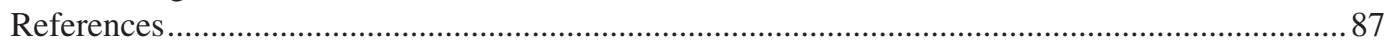

\section{INTRODUCTION}

Today, perhaps the most dynamically expanding branches of science are genetics and molecular and cellular biology, which are amassing impressive reams of data well ahead of scientists' capacity to fully analyse it all. Computational biology is emerging gradually as a response to the challenge of big data analytics. Quantum biology, on the other hand, is positioning itself to explain not so much the huge amounts of data but, like physics a century ago, a limited number of important, yet poorly understood phenomena such as photosynthesis, bioenergetics, vision, olfaction and bird navigation. Yet, the grandest challenge of all is to explain how the human brain works and, in particular, how conscious behaviour emerges from the structure and function of the human brain and its cellular and sub-cellular components. In this chapter, I revisit some historical issues in the development of quantum physics and then look at the emergence of quantum biology and finally quantum consciousness, aiming to provide new insights into these topics from both a physical and a historical perspective.

The birth of modern physics can be traced back to the reluctant introduction of what turned out to be a fundamental constant of nature, the so-called Planck constant, i.e. $h=6.6 \times 10^{-34} \mathrm{Js}$. Its introduction in an energy quantisation formula was the only solution to the problem of the heat emission spectra experimentally determined for physical objects at various temperatures. Max Planck postulated that these spectra originate from a discrete nature of energy levels in physical systems that can be quantised by the general relation:

$$
E_{n}=(n+1 / 2) h f
$$

where $n$ enumerates the energy levels and $f$ is the characteristic frequency of internal oscillations. This led to a revolutionary transformation of physical principles departing from the mechanistic laws of Newtonian physics to provide wave function descriptions of quantum physics that were developed in the decades that followed. In quantum physics, objects possess both a wave aspect and a particle aspect, a view of the physical world known as the principle of wave-particle duality, or 
complementarity. According to the Copenhagen interpretation of quantum mechanics, all the information about a particle or a system of particles can thus be described in a wave-like manner that is denoted mathematically by a wave function, $\phi(x, t)$. However, wave functions behave like waves and can diffract, and interfere together forming superpositions, implying that quantum particles simultaneously exist in multiple spatial locations and states. When a measurement is made, one of the multiple states is chosen and the quantum superposition of states ends being reduced to a classical state in a process known as the collapse of the wave function.

While quantum mechanics was developed with elementary particles in mind, its subsequent applications extended its validity to systems of many particles such as those encountered in condensed matter physics, e.g. in the description of the conduction electron 'sea', excitons, magnons, polarons, polaritons, etc. These types of quantum properties of macroscopic physical systems are called collective excitations. A system of many particles under specific conditions cannot be separated into individual wave functions for each particle; rather, the system is described by a single wave function describing its collective behaviour. This physical property is called quantum coherence, and it is characterised by individual particles losing their separate identities so that the entire system acts as a whole. Particles that were once unified in a common quantum state remain physically connected even at a distance. As a consequence of the collective behaviour of many-body systems, hallmarks of quantum mechanics can be seen in the properties of macroscopic objects such as crystals or ferromagnets, even above room temperature. There are also more exotic direct manifestations of quantum behaviour in macroscopic systems such as superconductors (with no measurable resistance to electrical current and ideal diamagnetism) or superfluids (with no viscosity and infinite vorticity). However, these latter two examples have so far been limited to very low temperatures. The precise location of the boundary (in terms of both the object's size and ambient temperature) between quantum and classical regimes is still under debate.

\section{FROM QUANTUM CHEMISTRY TO QUANTUM BIOLOGY}

Extensions of quantum mechanics to chemical compounds and chemical reactions proved to be exceedingly successful, and an entire field of quantum chemistry was developed as a consequence. In order to understand the creation of chemical bonds, especially covalent bonds in which electrons are shared between atoms of a molecule, a quantum mechanical wave function was introduced into the formalism as one of the postulates of quantum mechanics. All chemistry, including biochemistry, is based on the creation and destruction of bonds between atoms and hence on quantum interactions. Hence, by extension the structural stability of all living systems, like non-living systems, depends on quantum states at the level of chemical bonds. However, the quantum effects are commonly claimed to be washed out due to decoherence taking place at scales larger than individual atoms or sub-atomic particles, at higher temperatures and in aqueous media, which provide a noisy environment for particle interactions. Thus, the likelihood of quantum states playing functional roles at macroscopic scales in 'warm, wet and noisy' biological systems seems problematic due to environmental decoherence effects. On the other hand, it is reasonable to expect that evolution, through the process of natural selection over billions of years of experimentation and countless parallel attempts of trial and error, may have solved the decoherence problem so that quantum states may be essential features of biological systems (Rosa and Faber, 2004).

Erwin Schrödinger, Niels Bohr, Werner Heisenberg, Eugene Wigner and their contemporaries all offered speculations about quantum effects in living systems, while Schrödinger's famous book What Is Life? (Schrödinger, 1944) actually paved the way for the birth of molecular biology in the 1950 s. What is still very much a mystery, however, is the perfect synchronisation of biological processes across spatial and temporal dimensions that connect the hierarchical organisational scales of a living system. Here, quantum mechanics may indeed come to the rescue, if only we could explain the required absence of decoherence. 
Unfortunately, biological systems are so large and complex compared to standard physical systems that it is hard to separate 'pure' quantum effects from a large number of essentially classical processes that are also present. Thus, there is plenty of scope for disagreement about the extent to which life utilises non-trivial quantum processes. Given that the basic processes of biology take place at a molecular level, employing quantum effects for greater efficiency does not seem a priori implausible. Quantum coherence, collective modes of excitation and condensation phenomena, also offers attractive features that could shed light on the mechanisms of robustness and integrity of biological organisms. However, it is reasonable to expect that some of life's processes have evolved to the 'quantum edge', where a compromise may be struck between speed and accuracy. Since both physics and chemistry crucially depend on the power of quantum mechanics to provide fundamental insights into the world around us, it is natural to inquire whether biology offers examples of phenomena where quantum mechanics is the only viable explanation. This is indeed becoming increasingly clear, although examples of quantum effects in biology can so far be considered only a minor part of life processes as we know them.

G. Albrecht-Buehler (1992) found by clever experimentation that living cells perceive infrared electromagnetic waves with the peak of their sensitivity close to the wavelength of $1,000 \mathrm{~nm}$. He hypothesised that mitochondria, by proton transfer involved in energy production, release photons. Conversely, centrioles, dubbed by him the eye of the cell, are intricately structured to absorb these photons and trigger a signalling cascade. G. Albrecht-Buehler has been advocating a theory of cell functioning based on his conviction that the centriole plays the key role in orchestrating cellular activities by being both an eye and a brain of the cell. Cell movement is not random but directed and intentional. This is a crucial characteristic that distinguishes living from non-living matter. Cells control the movement of every part of their body. Furthermore, various parts of the cell can be likened to parts of the human body in their functional roles. Plasma membrane and cortex correspond to the skin and the musculature of a cell, which consists of small autonomously moving 'microplasts'. Their autonomy implies that cells contain a control system preventing the autonomous units from moving independently and randomly. The bulk cytoplasm including the mitochondria, organelles and intermediate filaments comprises the actual cell body excluding the nucleus, and corresponding to the 'guts' and 'innards' of the cell body. Its main cytoskeletal components are the intermediate filaments although microtubules (MTs) traverse this compartment everywhere. MTs mediate between the control centre (the centriole) and the autonomous domains. The control centre detects objects and other cells by pulsating near-infrared signals. Cells have structures functioning essentially as their 'eyes' in the form of centrioles. They are able to detect infrared signals and steer the cell movements towards their source. Evidence has been put forward that the signal detection is strongly localised in a narrow band of the near-infrared spectrum. If cells can detect light sources and measure space and time variables such as angles, distances, curvatures or durations, they must be able to derive these abstract quantities from the physical objects or signals of their environment. In response to exogenous signals, the centrosome may send destabilising signals along its radial array of MTs. The observed destabilisation is the signal that is propagated along the MTs like along nerves.

Engel et al.'s 2007 study of photosynthesis was a game-changer in the emerging field of quantum biology. Photosynthesis is a highly complicated and sophisticated mechanism that harvests light energy to split water by using individual photons to create a cascade of reactions. The process is extraordinarily efficient and represents a classic example of how evolution has fine-tuned the design of a physical system to attain near-optimal performance. The primary receptor of the light energy is a complex of pigment molecules known as chromophores that can become excited, and pass on the energy of excitation in a multi-stage process to the final reaction centre where charge separation occurs. Because the wavelength of the photon is much larger than the molecular assemblage, a superposition state of many excited pigment molecules is initially created and proceeds to evolve over a timescale of some hundreds of femtoseconds. Fleming and his group (Engel et al., 2007) used laser excitation and probe pulses to study the relaxation pathways of these light-harvesting 
complexes and observed a 'quantum beating' effect in which the maximum amplitude of the excitation visits and revisits different molecules in the system coherently. Fleming claims that, with appropriate timing, the system can 'grab' the coherent excitation (which persists for a few $100 \mathrm{fs}$ ) with greater probability than if it was merely distributed according to classical statistical mechanics. He believes this could lead to a multiplying of the speed of the energy transfer. An important feature of photosynthesis is that the molecular architecture involved is structured in a highly unusual and compact manner, suggesting it has been 'customised' to exploit long-range quantum effects (Blankenship and Engel, 2010). It could be that the particular configuration is efficient at preserving coherence for surprisingly long durations, enabling the system to 'explore' many pathways simultaneously and thus speed up a 'solution' (i.e. delivering energy to the reaction centre). In the light-sensitive complexes, reaction centres capture individual photons and transfer exciton energy by tunnelling avoiding decoherence even at room temperatures, which has been invoked on numerous occasions as a serious impediment to quantum biology (Tegmark, 2000) but also defended on various grounds (Hagan et al., 2002).

Beck and Eccles (1992) argued that the process of neurotransmitter release in the functioning of synapses is governed by the quantum uncertainty principle and involves quantum tunnelling. They further suggest that the introduction of quantum indeterminacy into neurotransmitter release mechanisms would allow for human free will of action. Their notion is that a quantum process, such as an electron tunnelling through an energy barrier, triggers exocytosis. The sheer size of the vesicle and the large number of neurotransmitter molecules contained in it make it next to impossible to lend itself to quantum tunnelling processes. Although the Beck-Eccles model contains very attractive ideas, the crux of the theory is incompatible with the present-day molecular biology of vesicular neurotransmitter release (Smith, 2009). Lowenstein (2000) made a powerful argument for the usefulness of quantum processes in receptor functions involving molecular recognition. All sensory inputs depend on this type of activity (olfaction, vision, sound, touch), and they all involve single molecules being triggers for amplification of these signals up to the neuron level and eventual brain activation. This amplification mechanism of the quantum signalling connects the microscopic and macroscopic levels which is critical to our understanding of the binding problem.

At the level of organs and tissues, it has been demonstrated that the human eye is capable of detecting light at an extremely low threshold, perhaps as few as two to three photons at a time (Hecht et al., 1942). Similarly, recent work by L. Turin, M. Stoneham and collaborators (Brookes et al., 2007) has provided strong support to the claim that the sense of smell (olfaction) is based on a quantum resonant energy transfer mechanism involving vibrational degrees of freedom of aromatic molecules and receptors in the membranes of olfactory nerves.

Special attention must be paid to the structural hierarchical organisation of biological systems, which in turn translates into an interlocking hierarchy of timescales. Faster timescales may inform processes at slower timescales about rapid processes taking place at a small spatial level. Amazingly, neural rhythms operate on timescales that vary from milliseconds to seconds, synchronise the forebrain and are mediated by neurotransmitter systems such as acetylcholine, norepinephrine and serotonin (Woolf et al., 2010). The neurotransmitter systems further fluctuate according to endogenous, circadian rhythms that also fluctuate according to the season of the year, which ultimately leads to an enormous range of timescales spanning between eight and ten orders of magnitude. Since neural events at the millisecond timescale can affect neural states at the circadian level, by extension it is entirely possible that quantum states at the picosecond scale could affect neural activity at the millisecond scale and above. Coupling between scales and amplification effects may offer a solution to some of these issues.

\section{TOWARDS QUANTUM CONSCIOUSNESS}

Does study of consciousness belong in the realm of natural sciences or is it a philosophical or even metaphysical area of inquiry? These questions have been pondered by many scientists, philosophers and spiritual leaders whose opinions diverge largely due to the subjective nature of consciousness. 
Obviously, the existence of this phenomenon cannot be denied as we all experience it as sentient humans. The author of this chapter firmly subscribes to the point of view that not only is consciousness a valid topic for scientific research but, in fact, it is also one of the most important unsolved scientific problems of our time. The problem of consciousness has defied conventional approaches, which view the brain as a classical computer, with neurons and synapses playing the roles of bit states. Specifically, the following enigmatic features remain unexplained: (1) the 'hard problem' of the nature of conscious experience, for example addressing the experience of 'qualia', our inner life; (2) the binding of disparate brain processes into unified concepts, objects and sense of self; (3) transition from preconscious processes to consciousness itself; (4) free will, or non-algorithmic (e.g. intuitive) processes; (5) the subjective flow of time; and (6) non-locality, i.e. paranormal connections between humans and also between humans and non-human species across large spatial and temporal domains (e.g. precognition, telepathy). Conventional neuronal-level computational approaches suggest conscious experience 'emerges' at a critical level of computational complexity. Binding is proposed to be accounted for by temporal synchrony (e.g. coherent 40-Hz oscillations) but, with no sense of the nature of conscious experience, temporal synchrony is merely correlative rather than explanatory. Perhaps the most potentially tractable problem is the transition from preconscious processes to consciousness itself. It is generally agreed upon that the vast majority of brain processes are non-conscious and that consciousness is the tip of an iceberg of brain activity. However, no specific brain area houses consciousness; neural activity in a given area may be non-conscious at one moment and correspond with consciousness at another. The classical approach suggests a critical level of computational complexity results in the transition via the emergence of consciousness, but again no threshold, biological correlate nor testable prediction has been put forth. Free will, subjective time flow and non-locality have not been seriously addressed by conventional approaches. Another shortcoming of conventional approaches is that neurons and synapses are considered as simple switches, whereas real biological cells are far more complex. For example, single-cell organisms such as paramecium swim, avoid obstacles and predators, learn, and find food and mates, all without possessing a single synapse. These cognitive functions can potentially be accomplished by the cell's cytoskeletal structures, primarily MTs.

Perhaps the first attempt to describe the brain using the terminology of quantum physics was made by Ricciardi and Umezawa (1967). Based on experimental observations of brain activity, they proposed that the brain could be conceived of as a spatially distributed system placed into particular quantum states by stimuli from the external environment. Thus, information can be thought of as being coded into the brain in the form of metastable excited states representative of short-term memory. This code would then be later on transferred to the ground state of the system by in the manner of Bose-Einstein condensation, which would account for learning and long-term memory. This model proposes that brain functions are manifestations of spontaneous symmetry breaking in the dynamics of the brain and regulated by long-range correlations. The model put forth by Ricciardi and Umezawa (1967) relating macroscopic quantum states to brain function, specifically memory, was later extended by these authors who proposed that the brain is a mixed physical system. In this model, the brain is considered to consist of two distinct interacting parts, the first part where the classical electrochemical interactions of the neurons of the brain occur, and the second being the macroscopic quantum state responsible for the creation and maintenance of memory.

R. Penrose $(1989,1994)$ hypothesised that quantum effects play a fundamental role in human consciousness by enabling the brain to perform non-computable computations. In his explanation of how the new physics can explain the mind and consciousness, he examined the division between classical and quantum physics, specifically the measurement problem, and related the collapse of the wave function to conscious events using the notion of objective reduction. This led to the suggestion that MTs within neurons provide the brain with structures capable of orchestrating the collapse of the wave function via quantum computations. This combination has become known as the Penrose-Hameroff orchestrated objective reduction (Orch OR) theory (Hameroff, 1998; Hameroff and Penrose, 2014). The basic idea on which Orch OR rests is that MTs within the brain's neurons 
function as quantum computers, with MT protein subunits (tubulins) existing transiently in quantum superposition of two or more conformational states (i.e. as quantum bits, or 'qubits'). The quantum state reductions yield conscious perceptions and volitional choices, which then govern neuronal actions. This is essentially the same idea on which technological quantum computing is based, except that in Orch OR the proposed qubits are tubulin protein conformations, and the reduction/ collapse occurs due to a specific objective threshold (objective reduction) rather than environmental interaction. The theory considers a conscious event as a quantum computation, which concludes via objective state reduction. The biological conditions in the brain, including synaptic activity, are considered to influence the quantum computations, thus orchestrating the collapse of the qubits and giving rise to a conscious event. 'Orch OR' is an attempt to place consciousness within the empirical sciences as a fundamental concept in science. The central postulate of the Orch OR theory is that the site of action of consciousness is located within the brain's MTs which operate at the interface between classical neurophysiology and quantum gravitational forces. These are very bold claims that have found both ardent supporters (Stapp, 1995) and vocal critics (Seife, 2000) in the scientific community.

There have been many debates concerning whether the quantum description of consciousness is valid, realistic or needed. However, only recently have advances in nanotechnology been made, allowing for serious empirical investigation into the biophysical workings of sub-cellular structures. As such, the lack of evidence in support of quantum brain theories should not be taken as proof against these theories, but rather as an area in need of careful and vigorous scientific investigation. The several enigmatic features of consciousness are still, for the most part, left unexplained by classical theories. The apparent ability of quantum theories to answer these questions may provide new avenues of investigation into consciousness. Macroscopic quantum phenomena such as superconductivity, and superfluidity need to be highly isolated from their environment in order to avoid the effects of decoherence. In order for such phenomena to exist in the brain, nature would need to provide mechanisms to isolate against decoherence. Clearly, these issues are not completely resolved. Thus, investigations into the quantum nature of MTs are still badly needed.

Empirically, a host of studies indicate that the MT matrix in dendrites is structurally reorganised with learning and memory. Using an associative learning paradigm combined with immunohistochemistry, fear conditioning either to tone or to the training context induced significant changes in MT-associated protein (MAP2) in circumscribed regions of the cerebral cortex or hippocampus, with alterations correlating with the type of training (Woolf et al., 1994, 1999). In terms of molecular biophysics, based on their ability to propagate signals through the neuron, MTs and actin filaments can be viewed as computationally relevant nanowire networks that operate within neurons (Woolf et al., 2010). Rather than inputs to neurons being limited to causing discrete responses, this viewpoint offers the possibility of local and global neuroplasticity, based on the cytoskeleton computing and storing templates that translate patterns of inputs across widespread synapses into the 'behavioural' output of the neuron. This behavioural output of the neuron is not limited to axonal firing and dendritic integration of electrochemically mediated inputs. Instead, it includes connecting the cell nucleus with the postsynaptic density, initiating transport of receptor molecules, membrane proteins, organelles and mRNA, regulating neurite motility, restructuring of spines and complex dendrite architecture, the lateral movement of receptor and membrane proteins of neurons, and governing the availability of ion channels in the membrane.

\section{FUTURE OUTLOOK}

I foresee major progress in bridging the gap between nanoscience and consciousness in the area of nano-neuroscience where MTs, actin filaments and motor proteins connect between neurophysiology and molecular biology. Studying neural phenomena at a nanoscale could lead to monumental breakthroughs in science and medicine and aid in consciousness studies. Further possibilities involving physically based quantum mechanisms of consciousness should also be considered. The 
basic idea is to investigate if there are other quantum network architectures that could be operating in the brain. First of all, quantum entanglement in such a network could provide at least a partial answer to the binding problem of consciousness allowing for a delocalised quantum state involving many neurons. This requires a thorough understanding of quantum networks. It is worth emphasising that quantum networks may lead to quantum memories, whereby entangled states store information such as visual inputs. Moreover, quantum networks could generate communication channels that would transport information and process it performing complex operations. Quantum computation in the brain (Litt et al., 2006) would surely be beneficial from an evolutionary standpoint, and biology has had 4 billion years to solve the decoherence problem.

Recently, an interesting proposal was put forward suggesting direct involvement of nuclear spin in long-lived quantum states attributed to consciousness in the human brain (Fisher, 2017). However, several challenging issues remain to be addressed. First of all, due to thermal fluctuations, a magnetic field of sufficient strength would be required to prepare the spin system in a pure enough state. On the other hand, there are no naturally occurring large magnetic fields and we also know that strong magnetic fields such as in magnetic resonance imaging (MRI) machines do not have a significant effect on the state of consciousness of the person subjected to MRI scans. Regarding quantum communication channels, photon emission and absorption is the best candidate mechanism for such phenomena. Biophotonics is an emerging field in spite of its long history of false starts and intermittent periods of dormancy. A recent review (Cifra and Pospíšil, 2014) summarises the landscape in this field, emphasising a relatively narrow range of wavelengths playing a role in biophotonics, namely between 350 and $1,300 \mathrm{~nm}$. The generation of photons inside living cells is mainly related to recombination of reactive oxygen species. It is also interesting to consider signal amplification and transmission over macroscopic distances along axons and dendrites of neurons. The tenuous connection between quantum biology, consciousness and electromagnetic fields, if properly supported by precise experimental investigations, could become a nexus for rigorous explorations of how our brain operates beyond the confines of conventional neuroscience.

Understanding the biological basis for sustained quantum coherent superposition and entanglement would not only help solve the enigmatic features of consciousness, but also enable future quantum information technologies.

\section{ACKNOWLEDGEMENTS}

The author acknowledges funding support for his research from NSERC (Canada).

\section{REFERENCES}

Albrecht-Buehler, G. (1992) Rudimentary form of cellular "vision". Proc. Natl. Acad. Sci. U. S. A. 89: 8288-8292.

Beck, F. and Eccles, J.C. (1992) Quantum aspects of brain activity and the role of consciousness. Proc. Natl. Acad. Sci. U. S. A. 89: 11357-11361.

Blankenship, G.R. and Engel, G.S. (2010) Long-lived quantum coherence in photosynthetic complexes at physiological temperature. Proc. Natl. Acad. Sci. U. S. A. 107(29): 12766-12770.

Brookes, J.C., Hartoutsiou, F., Horsfield, A.P., Turin, L., and Stoneham, A.M. (2007) Could humans recognize odor by phonon assisted tunneling? Phys. Rev. Lett. 98(3): 038101.

Cifra, M. and Pospíšil, P. (2014) Ultra-weak photon emission from biological samples: Definition, mechanisms, properties, detection and applications. J. Photochem. Photobiol. B Biol. 139: 2-10.

Engel, G.S., Calhoun, T.R., Read, E.L., Ahn, T.K., Mancal, T., Cheng, Y.C., Blankenship, R.E. and Fleming, G.R. (2007) Evidence for wavelike energy transfer through quantum coherence in photosynthetic systems. Nature 446(7137): 782-786.

Fisher, M.P. (2017) Are we quantum computers, or merely clever robots. Int. J. Mod. Phys. B 31(07): 1743001. Hagan, S., Hameroff, S.R., and Tuszynski, J.A. (2002) Quantum computation in brain microtubules: Decoherence and biological feasibility. Phys. Rev. E 65(61901): 1-10. 
Hameroff, S. (1998) Quantum computation in brain microtubules? The Penrose-Hameroff 'Orch OR' model of consciousness [and discussion]. Phil. Trans. R. Soc. London A 356(1743): 1869-1896.

Hameroff, S. and Penrose, R. (2014) Consciousness in the Universe: A review of the "Orch OR" theory. Phys. Life Rev. 11(1): 39-78.

Hecht, S., Shlaer, S., and Pirenne, M.H. (1942) Energy, quanta, and vision. J. Gen. Physiol. 25: 819-840.

Litt, A., Eliasmith, C., Kroon, F.W., Weinstein, S., and Thagard, P. (2006) Is the brain a quantum computer? Cognit. Sci. 30: 593-603.

Lowenstein, W. (2000) Touchstone of Life. Oxford: Oxford University Press.

Penrose, R. (1989) The Emperor's New Mind. Oxford, New York and Melbourne: Oxford University Press.

Penrose, R. (1994) Shadows of the Mind. Oxford, New York and Melbourne: Oxford University Press.

Ricciardi, L.M. and Umezawa, H. (1967) Brain and physics of many-body problems. Kybernetik 4(2): 44-48.

Rosa, L.P. and Faber, J. (2004) Quantum models of the mind: Are they compatible with environment decoherence? Phys. Rev. E. 70: 031902.

Schrödinger, E. (1944) What Is Life? Cambridge: Cambridge University Press.

Seife, C. (2000) Cold numbers unmake the quantum mind. Science 287(5454): 791.

Smith, C.U. (2009) The 'hard problem' and the quantum physicists. Part 2: Modern times. Brain Cogn. 71: 54-63.

Stapp, H. (1995) Why classical mechanics cannot naturally accommodate consciousness but quantum mechanics can. Psyche 2(5): 1-23.

Tegmark, M. (2000) Importance of quantum coherence in brain processes. Phys. Rev. E 61, 4194-4206.

Woolf, N.J., Young, S.L., Johnson, G.V., and Fanselow, M.S. (1994) Pavlovian conditioning alters cortical microtubule-associated protein-2. Neuroreport 9: 1045-1048.

Woolf, N.J., Zinnerman, M.D., and Johnson, G.V. (1999) Hippocampal microtubule-associated protein-2 alterations with contextual memory. Brain Res. 6: 241-249.

Woolf, N.J., Priel, A., and Tuszynski, J.A. (2010) Nanoneuroscience: Structural and Functional Roles of the Neuronal Cytoskeleton in Health and Disease. Heidelberg: Springer Verlag. 